\title{
Correction to: Retraction of: Tumor Protein D52-Like 2 Accelerates Gastric Cancer Cell Proliferation; 10.10.89/cbr.2014.1766 Cancer Biother Radiopharm 2017;32(10):388. DOI: 10.1089/cbr.2014.1766.retract
}

Tn the December 2017 issue of Cancer Biotherapy and Radiopharmaceuticals (vol. 32, no. 10, page 388), there were errors 1 requiring correction in the retraction notice entitled, Tumor Protein D52-Like 2 Accelerates Gastric Cancer Cell Proliferation; 10.10.89/cbr.2014.1766.

\section{List of corrected text in this retraction:}

Original retraction text: ... this study employed the 5'-GCGG... shRNA sequence as a "TPD52L2 targeting sequence", despite the fact that this sequence shows no homology with any TPD52L2 sequence, or indeed any sequence in the nonredundant nucleotide database.

- CORRECTED text: ... this study employed the 5'-GTAG... shRNA sequence as a "negative control," despite the fact that according to Blastn analyses, this sequence shows homology to the human NOB1 gene.

Original retraction text: ... since the article did not employ a lentiviral construct targeting the TPD52L2 gene as described in the study, it most likely invalidates the experimental results reported in the article.

- CORRECTED text: ... since the article did not employ a non-targeting lentiviral construct as described in the study, it most likely invalidates the experimental results reported in the article.

The online version of the retraction notice has been corrected to reflect this change. 